\title{
TAMANHO CORPORAL EM POPULAÇÕES NATURAIS DE Didelphis (MAMMALIA: MARSUPIALIA) DO SUL DO BRASIL
}

\author{
CÁCERES, N. C. e MONTEIRO-FILHO,E. L. A. \\ Departamento de Zoologia, Universidade Federal do Paraná, C.P. 19020, CEP 81531-970, Curitiba, PR \\ Correspondência para: Nilton C. Cáceres, Departamento de Zoologia, Universidade Federal do Paraná, C.P. 19020, \\ CEP 81531-970, Curitiba, PR, e-mail: ncaceres@garoupa.bio.ufpr.br \\ Recebido em 09/07/97 - Aceito em 04/09/98 - Distribuído em 10/09/99
}

(Com 3 figuras)

\section{ABSTRACT \\ Body size in natural populations of Didelphis (Mammalia:Marsupialia) from southern Brazil}

Nowaday, data about marsupial biometry are poorly available in Brazil, considering the high richness of marsupial species in this country. Then, relationships of body size and sexual dimorfism of natural populations of the white-eared opossum, Didelphis albiventris, and of the common opossum, D. marsupialis, were studied in two mixed forests of Curitiba, southern Brazil, between February 1995 and February 1997. As a result, there was a strong relationship among the teeth emergence, weight and several body measures obtained from opossums. Hence, measures as the head length may be used to estimate other measures of the body. Sexual dimorfism was also verified in relation to the body size and the head shape for the common opossum. Adult females of the white-eared opossum proved to be bigger than adult females of the common opossum. Besides, some individuals of both species had an increase in their weight during the autumn probably to support the low food available' season. However, the white-eared opossum usually storages more fat that the common opossum in any season. Therefore, the adaptative strategies of both species revealed different among themselves in southern Brazil and probably are results of different life histories, despite their related kinship.

Key words: opossums, biometry, growth, sexual dimorfism, fat index.

\section{RESUMO}

Atualmente, poucos são os dados biométricos de marsupiais disponíveis no Brasil considerando-se sua riqueza de espécies. Baseando-se nisto, as relações de tamanho corporal de populações naturais do gambáde-orelha-branca, Didelphis albiventris, e do gambá-de-orelha-preta, D. marsupialis, foram verificadas em dois remanescentes florestais de Curitiba, Sul do Brasil, entre fevereiro de 1995 e fevereiro de 1997. Como resultado, houve uma forte relação entre a erupção de dentes, o peso e a maioria das medidas corporais obtidas de ambas as espécies. Em conseqüência, parâmetros como o comprimento da cabeça podem ser utilizados para estimar os demais parâmetros corporais dos marsupiais estudados. Foi também observado dimorfismo sexual quanto ao tamanho corporal e formato da cabeça para D. marsupialis. Comparativamente, as fêmeas adultas de $D$. albiventris apresentam maior tamanho que as fêmeas adultas de D. marsupialis, pelo menos no Sul do Brasil. Houve evidências de que alguns indivíduos podem ter um aumento em seu peso corporal durante o outono, talvez como uma maneira de suportar a estação com menor disponibilidade de alimentos. Por outro lado, D. albiventris armazena gorduras mais freqüentemente, independente da estação do ano. Assim, as estratégias adaptativas de ambas as espécies de marsupiais quanto ao tamanho corporal no Sul do Brasil apresentaram algumas diferenças e provavelmente são resultantes de modos de vida também diferentes, apesar de seu parentesco próximo.

Palavras-chave: gambás, biometria, crescimento, dimorfismo sexual, índice de obesidade. 


\section{INTRODUÇÃO}

O gambá-de-orelha-preta, Didelphis marsupialis L., 1758, e o gambá-de-orelha-branca, $D$. albiventris (Lund, 1841), são espécies de marsupiais didelfídeos extensamente distribuídas na Região Neotropical (Emmons \& Feer, 1990).

Apesar de suas amplas distribuições, poucas são as informações sobre tamanho corporal desses marsupiais, principalmente no Brasil, onde existe pelo menos uma das duas espécies em cada bioma (Emmons \& Feer, 1990).

Nos neotrópicos, as informações sobre tamanho corporal de populações de Didelphis concentram-se basicamente em estudos como os de Gardner (1973), Tyndale-Biscoe \& Mackenzie (1976), Varejão \& Valle (1982), Atramentowicz (1986), Monteiro-Filho (1987), Fonseca \& Kierulff (1988), Cherem et al. (1996) e Catzeflis et al. (1997), sendo que apenas poucos desses tratam de espécies simpátricas de marsupiais. Assim, há a necessidade de estudos que envolvam a biometria dessas espécies e suas implicações ecológicas.

Procurando fornecer informações biométricas adicionais para as espécies de marsupiais didelfídeos, o objetivo deste estudo consiste na obtenção de dados sobre tamanho corporal provenientes de populações naturais de $D$. albiventris e de $D$. marsupialis em dois fragmentos florestais de uma área urbana do Sul do Brasil.

\section{ÁREAS ESTUDADAS}

Localizam-se em região urbana da cidade de Curitiba, Estado do Paraná $\left(25^{\circ} 25^{\prime} \mathrm{S}\right.$ e $\left.49^{\circ} 18^{\prime} \mathrm{W}\right)$, aproximadamente 940 metros acima do nível do mar. Uma dessas áreas pertence à Companhia Paranaense de Energia (Copel), com 5 ha, na qual Didelphis albiventris e D. marsupialis ocorrem simpatricamente. A segunda área estudada, com 2,5 ha, pertence ao Centro Politécnico da Universidade Federal do Paraná (UFPR) e fica a $8 \mathrm{~km}$ da Copel. Nessa última área, ocorre somente $D$. albiventris. Ambos os locais possuem remanescentes com floresta ombrófila mista alterada, circundada por estradas de rodagens, residências, prédios e terrenos baldios. A temperatura média anual da cidade oscila em torno de $16,5^{\circ} \mathrm{C}$ e a umidade relativa do ar é geralmente elevada, com os meses mais secos do ano concentrando- se principalmente no outono e inverno (Maack, 1981). Há duas estações não tão bem definidas na região, uma mais seca (abril-agosto) e outra mais úmida (setembro-março) (dados da Estação Meteorológica da Universidade Federal do Paraná).

\section{MATERIAL E MÉTODOS}

A obtenção de dados biométricos de ambas as espécies de marsupiais em estudo estendeu-se por 12 meses na área da Copel (fevereiro de 1995 a janeiro de 1996), nos quais 30 gaiolas de capturas $(20 \times 20 \times 40 \mathrm{~cm})$ foram dispostas de maneira uniforme e em pontos fixos ( $\cong 40 \mathrm{~m}$ entre cada gaiola). Essas foram armadas uma vez por semana, sempre de tarde, e verificadas na manhã seguinte. $\mathrm{Na}$ área da UFPR, 10 gaiolas de capturas $(40 \times 20 \times 20$ $\mathrm{cm}$ ) foram armadas no local duas vezes por mês durante quatro meses (novembro de 1996 a fevereiro de 1997). A isca utilizada em ambas as áreas estudadas foi banana com óleo de fígado de bacalhau. Todos os indivíduos capturados foram marcados por combinações de orifícios nas orelhas (Monteiro-Filho, 1987), mensurados, pesados, sexados e tiveram suas idades estimadas (ver Gilmore, 1943; Tyndale-Biscoe \& Mackenzie, 1976; Atramentowicz, 1986) antes de serem liberados. As informações sobre tamanho corporal, como o comprimento da cabeça (extremidade do focinho à base do côndilo-occipital), tronco (base do côndilo-occipital à base da cauda), cauda, orelha e pata posterior (exclusive a unha), foram obtidas através de mensurações feitas com uma trena metálica (precisão de $0,5 \mathrm{~mm}$ ). A largura da cabeça na altura do arco zigomático também foi medida, porém com um paquímetro de precisão de $0,1 \mathrm{~mm}$. O peso foi verificado por meio de um dinamômetro com capacidade máxima de $3 \mathrm{~kg}$ (precisão de $5 \mathrm{~g}$ ).

Os pesos de fêmeas com filhotes foram estimados subtraindo-se o peso da ninhada (Hossler et al., 1994). Todos os parâmetros obtidos foram confrontados entre si por meio de análises de correlação, depois de agrupá-los mensalmente para as análises.

A fim de comparar as taxas de crescimento inter e intra-especificamente, curvas de crescimento foram elaboradas utilizando-se mensurações de indivíduos recapturados mensalmente. 
Com a finalidade de verificar um eventual dimorfismo sexual, as proporções de largura/comprimento das cabeças mensuradas foram analisadas intersexualmente em cada espécie de marsupial através da Análise de Variância (Zar, 1984). Para a análise, foram utilizados indivíduos adultos de D. marsupialis e adultos e jovens-subadultos de D. albiventris (cada grupo tratado separadamente). Para efeito de comparação, as informações biométricas de Varejão \& Valle (1982) quanto à relação largura/comprimento do crânio de indivíduos adultos de $D$. marsupialis foram também analisadas. Os demais parâmetros corporais mensurados foram também submetidos à Análise de Variância (Zar, 1984), com o objetivo de detectar outras formas de dimorfismo.

Para obter um índice de obesidade nos diferentes períodos do ano, o peso de cada animal foi dividido pelo tamanho de sua cabeça, pressupondo-se que este último parâmetro esteja diretamente relacionado ao tamanho do animal, não sofrendo variações influenciadas por falta de alimento ou por mutilações.

\section{RESULTADOS}

$\mathrm{Na}$ área da Copel, foram capturados 18 indivíduos de D. marsupialis, 7 machos e 11 fêmeas, totalizando 172 capturas (das quais em 60 delas foram verificados todos os parâmetros corporais e, no restante, apenas o peso, dentição e número de registro), assim como 20 indivíduos de $D$. albiventris, 9 machos e 11 fêmeas, totalizando 60 capturas (das quais em 26 delas foram verificados todos os parâmetros e, no restante, apenas o peso, idade e sexo). $\mathrm{Na}$ área da UFPR, foram capturados 17 indivíduos de D. albiventris, sendo 7 machos e 10 fêmeas, em um total de 22 capturas.

Os parâmetros corporais mensurados mostraram-se bem correlacionados entre si para $D$. albiventris, exceção feita ao comprimento da orelha (Tabela 1).

Similarmente, para D. marsupialis, todos os parâmeros foram bem correlacionados (Tabela 1). Houve forte correlação entre as classes dentárias e os parâmetros estudados (Tabela 2).

Foi constatado que machos e fêmeas de $D$. albiventris e machos de $D$. marsupialis apresentam crescimento mais acelerado e maior tamanho quando adultos que fêmeas de D. marsupialis (Fig. 1, Tabela 2). Com base na dentição, a maioria dos indivíduos de ambas as espécies alcançou o estágio adulto dentro de 9 ou 10 meses após o nascimento. Da mesma forma, foram observadas evidências de que machos de ambas as espécies, usualmente, atingem mais cedo a condição de adultos (Tabela 2).

Através da Análise de Variância, foi constatado dimorfismo sexual em indivíduos adultos de $D$. marsupialis quanto à relação largura/comprimento da cabeça $\left(\mathrm{F}_{1,13}=5,82 ; \mathrm{p}<0,04\right)$, com fêmeas tendendo a terem cabeças mais estreitas que as dos machos. O mesmo ocorreu com os dados craniométricos de Varejão \& Valle (1982) $\left(\mathrm{F}_{1,26}=5,08 ; \mathrm{p}<0,04\right)$.

TABELA 1

Índices de correlação intra-específica entre as medidas de tamanho corporal de indivíduos do gambá-deorelha-branca, Didelphis albiventris, e do gambá-de-orelha-preta, D. marsupialis, do Sul do Brasil.

\begin{tabular}{|c|c|c|c|c|c|c|c|}
\hline & \multicolumn{7}{|c|}{ D. albiventris } \\
\hline & Cabeça-C & Cabeça-L & Tronco & Cauda & Orelha & Pata post. & Peso \\
\hline Cabeça-C & 1 & 0,97 & 0,95 & 0,86 & 0,46 & 0,97 & 0,98 \\
\hline Cabeça-L & 0,96 & 1 & 0,97 & 0,83 & 0,55 & 0,98 & 0,98 \\
\hline Tronco & 0,95 & 0,92 & 1 & 0,90 & 0,59 & 0,94 & 0,98 \\
\hline Cauda & 0,83 & 0,80 & 0,74 & 1 & 0,37 & 0,89 & 0,89 \\
\hline Orelha & 0,95 & 0,86 & 0,92 & 0,75 & 1 & 0,57 & 0,51 \\
\hline Pata post. & 0,94 & 0,80 & 0,94 & 0,77 & 0,94 & 1 & 0,96 \\
\hline \multirow[t]{2}{*}{ Peso } & 0,97 & 0,93 & 0,94 & 0,76 & 0,95 & 0,88 & 1 \\
\hline & \multicolumn{7}{|c|}{ D. marsupialis } \\
\hline
\end{tabular}




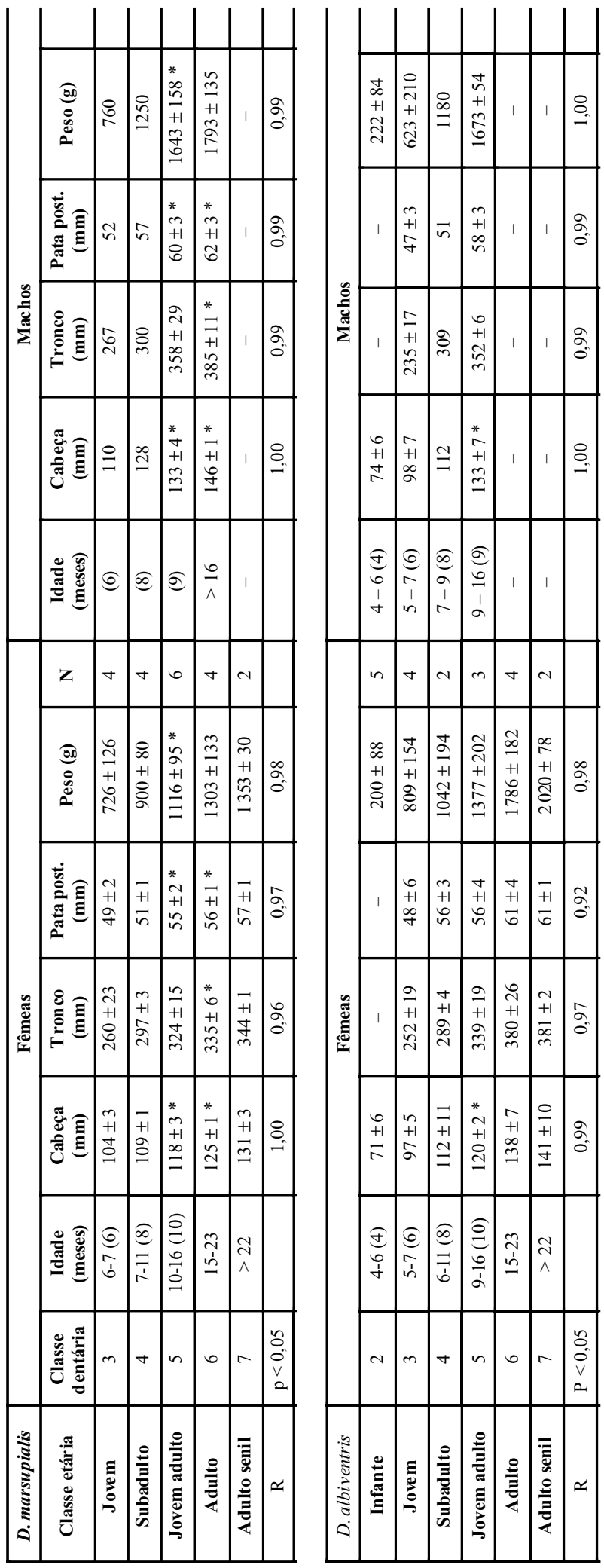




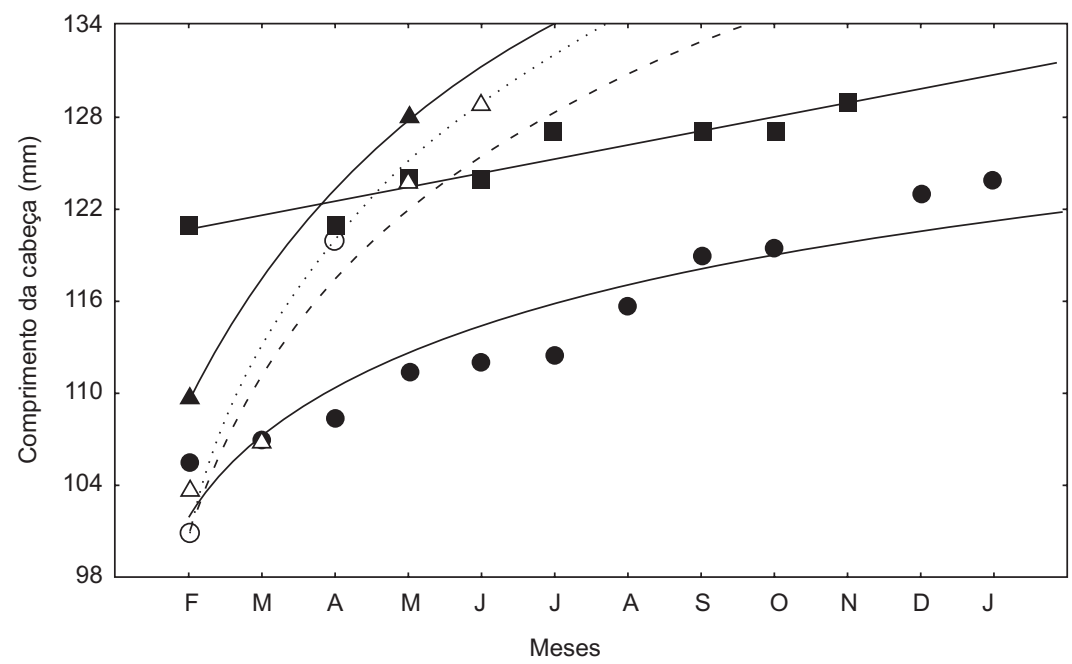

Fig. 1 - Curvas de crescimento de Didelphis albiventris (em branco com linha interrompida) e de D. marsupialis (em preto com linha contínua) capturados na área da Copel, cidade de Curitiba, durante 1995 e 1996 (D. albiventris - triângulo com linha tracejada indica um macho jovem $\bullet$ adulto, círculo com linha pontilhada indica uma fêmea jovem $\bullet$ subadulta; $D$. marsupialis - triângulo indica um macho jovem adulto, círculo indica média de três fêmeas jovens $\bullet$ adultas, quadrado: uma fêmea adulta).

Essas evidências são suportadas em $D$. marsupialis através de diferenças sexuais também significativas em outros parâmetros corporais analisados, como o comprimento da cabeça (Fig. 2), o comprimento da pata posterior e o peso (Tabela 2). Já para $D$. albiventris, inão foi detectado dimorfismo quanto ao formato da cabeça tanto para jovens-subadultos $\left(\mathrm{F}_{1,8}=0,07 ; \mathrm{p}>0,80\right)$ quanto para adultos $\left(F_{1,9}=2,72 ; p>0,13\right)$ (Tabela 2, Fig. 2). Quanto às demais medidas de D. albiventris, apenas o comprimento da cabeça de adultos mostrou-se significativamente diferente na comparação entre os sexos, embora machos adultos mais idosos não tenham sido amostrados (Tabela 2).

Foi observado que ambas as espécies, principalmente $D$. marsupialis, acumulam gorduras no corpo durante o outono e as utilizam durante o inverno, fato que foi possível observar com o ganho e posterior perda de peso dos animais, respectivamente, nesses períodos (Fig. 3). Observou-se também que indivíduos de $D$. albiventris, em geral, apresentam elevados índices de obesidade quando adultos (Fig. 3). Fêmeas mais idosas de D. albiventris apresentaram peso corporal de 500 a $700 \mathrm{~g}$ a mais que fêmeas de $D$. marsupialis de mesma idade (Tabela 2).

\section{DISCUSSÃO}

Baseando-se nos elevados coeficientes de correlação obtidos, as várias partes do corpo de Didelphis desenvolveram-se sob as mesmas taxas de crescimento, exceto o comprimento da orelha de $D$. albiventris e que se mostrou bem variável intrapopulacionalmente. Desse modo, pode-se obter um parâmetro corporal a partir do outro utilizando quaisquer dos parâmetros corporais analisados (ver Tyndale-Biscoe \& Mackenzie, 1976; MonteiroFilho, 1987).

Apesar das observações de Petrides (1949), o peso também constitui-se um bom parâmetro para estimar o tamanho corporal em Didelphis ainda não-adultos (Iskjaer et al., 1989). Como a erupção de dentes é bem correlacionada à idade em $D i$ delphis (Gilmore, 1943; Petrides, 1949; Gardner, 1973; Tyndale-Biscoe \& Mackenzie, 1976; Atramentowicz, 1986), pode-se estimar também a idade através dos parâmetros analisados. De maneira geral, essas informações são válidas para indivíduos não-adultos, pois não há relação entre esses parâmetros para adultos de idade avançada, pelo menos para D. marsupialis (Tyndale-Biscoe \& Mackenzie, 1976). 
As informações de vários autores (Gilmore, 1943; Petrides, 1949; Atramentowicz, 1986) corroboram nossas observações de que as espécies de Didelphis atingem o estágio adulto por volta de 9 ou 10 meses de idade, o que é um tempo relativamente curto, tendo em vista os mamíferos placentários (Thompson, 1987). Além disso, Fonseca et al. (1982) observou que uma fêmea de $D$. albiventris apresentou elevada taxa de crescimento, aumentando o seu peso em $258 \%$ em apenas 43 dias.

De acordo com Gardner (1973), corroborado por Pine et al. (1985), o fato de machos de D. mar- supialis apresentarem crescimento mais acelerado que as fêmeas deve estar relacionado ao fato que estes não apresentam gastos energéticos com a gestação e, principalmente, a lactação. Corroborando essa hipótese, Ryser (1992) em um estudo com $D$. virginiana Kerr, 1892, informou que o peso de machos tem elevada relação com seu sucesso reprodutivo, além do fato de alguns machos de $D$. marsupialis capturados em uma das áreas estudadas durante a estação reprodutiva encontrarem-se muito machucados (observação pessoal), talvez em conseqüência de disputas por acasalamentos entre eles.

Didelphis albiventris

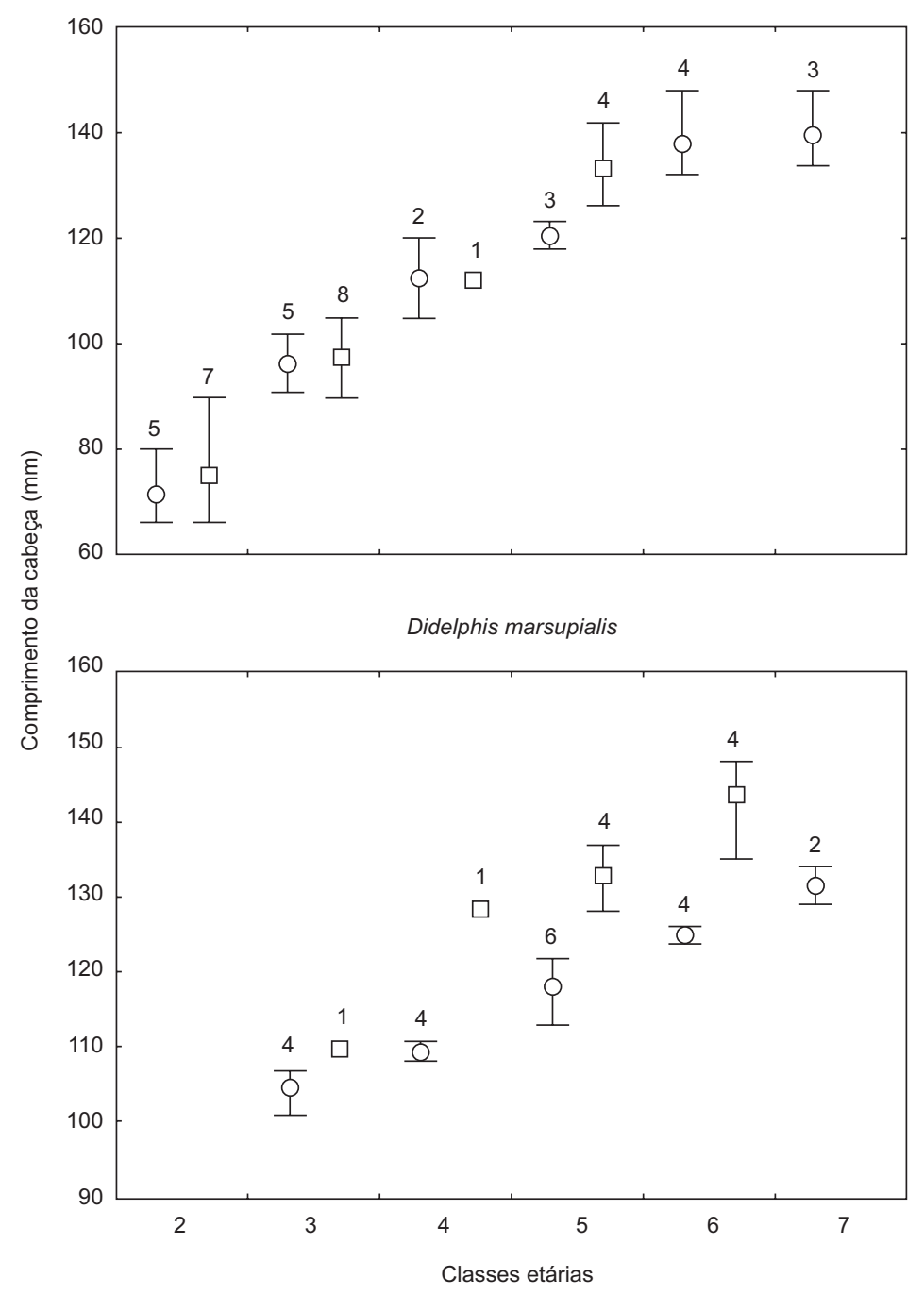

Fig. 2 - Comprimentos da cabeça de machos e fêmeas de Didephis, evidenciando dimorfismo sexual em uma das espécies. Machos indicados por quadrados e fêmeas, por círculos. Linhas verticais indicam a amplitude das medidas. Números acima dos pontos indicam o número de indivíduos amostrados. Classes etárias segundo Tyndale-Biscoe \& Mackenzie (1976). 


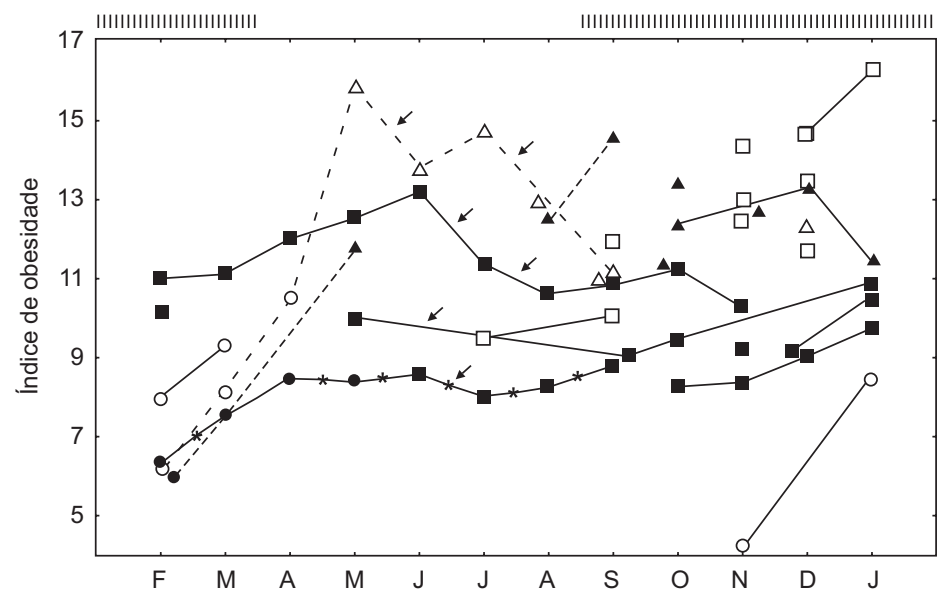

Fig. 3 - Índice de obesidade (proporção do peso em g/comprimento da cabeça em mm) de Didelphis albiventris (símbolos em branco) e D. marsupialis (símbolos em preto) obtidos em Curitiba entre 1995 e 1997. Símbolos isolados indicam indivíduos diferentes e símbolos unidos por linhas indicam os mesmos indivíduos. Linha com asteriscos indica média de três indivíduos de mesma idade. Quadrados indicam fêmeas adultas, triângulos indicam machos adultos e círculos indicam infantes/jovens/ subadultos (com dentição incompleta). Setas frisam a perda de peso durante a estação seca. Barras acima do gráfico indicam a estação úmida em Curitiba.

Similarmente ao nosso estudo, Petrides (1949), Gardner (1973), Tyndale-Biscoe \& Mackenzie (1976), Fonseca \& Kierulff (1988) e Cherem et al. (1996) constataram dimorfismo sexual em várias partes do corpo de Didelphis. Contudo, Atramentowicz (1986), na Guiana Francesa, constatou apenas diferença no peso de D. marsupialis.

Diferentemente das observações de Varejão \& Valle (1982) em Minas Gerais e de Catzeflis et al. (1997) na Guiana Francesa, as fêmeas de $D$. marsupialis não apenas se mostraram menos pesadas que as fêmeas de $D$. albiventris (pelo fato de acumularem gorduras no corpo menos freqüentemente - ver Gardner, 1983), mas também mostramse menores em relação ao tamanho corporal no Sul do Brasil. Possivelmente, não somente a seleção sexual influencia no tamanho corporal das espécies, mas também outros fatores, como a disponibilidade de alimentos (Gardner, 1973; Shine, 1989) e a latitude (Quin et al., 1996). Considerando as diferenças de tamanho corporal em espécies simpátricas de marsupiais, Leite et al. (1994) sugeriram que o tamanho corporal pode desempenhar um papel importante na partilha de recursos entre tais espécies.

O dimorfismo na forma da cabeça de D. marsupialis confirma as observações feitas por nós em campo. Fato este que é corroborado pelos dados obtidos por Gardner (1973) e Varejão \& Valle (1982) para D. marsupialis e por Monteiro-Filho (dados não-publicados) para $D$. albiventris do
Sudeste do Brasil. Por outro lado, tal dimorfismo não deve ter sido constatado aqui para $D$. albiventris devido à ausência de capturas de machos adultos de maior idade. Como foi comentado para Monodelphis dimidiata (Wagner) por Pine et al. (1985), dimorfismos acentuados podem resultar em separações de nicho ecológico entre os sexos (ver também Shine, 1989).

O dimorfismo observado em $D$. marsupialis poderia resultar em uma moderada separação de nicho alimentar entre machos e fêmeas, com os primeiros podendo se alimentar mais freqüentemente de itens maiores (como por exemplo roedores, ver Pine et al., 1985). Em auxílio a esta observação, Hershkovitz (1992) informou sobre dimorfismo sexual no aparato mastigatório de espécies marsupiais do gênero Gracilinanus. Outras evidências de tal separação de nicho alimentar foram observadas por Moors (1980) para mustelídeos.

O ganho de peso como forma de suportar épocas desfavoráveis foi observado por Fitch \& Sandidge (1953) e Charles-Dominique et al. (1981) para várias espécies de marsupiais didelfídeos, incluindo Didelphis. A rarefação de alimentos durante a estação seca e o maior gasto energético com o início da reprodução no final desta estação (Fonseca \& Kierulff, 1988; Cáceres \& Monteiro-Filho, 1998) talvez estejam relacionados com tal fenômeno. Esse aumento de peso deve ocorrer principalmente no tecido subepidérmico (ob- 
servação pessoal), controlado hormonalmente, como sugeriu Monteiro-Filho (1987) para D. albiventris. Porém, o ganho de peso inerente ao crescimento corporal ou mesmo por fenômenos como a lactação também ocorrem em Didelphis (Sunquist \& Eisenberg, 1993; Hossler et al., 1994).

Em resumo, as espécies de Didelphis do Sul do Brasil apresentaram padrões de crescimento corporal rápido e uniforme, dimorfismo sexual pelo menos para D. marsupialis e maior tamanho corporal para D. albiventris, fatos que são prováveis indícios de estratégias de vida diferentes entre essas espécies simpátricas de marsupiais, apesar de seu parentesco próximo.

Agradecimentos - Somos gratos a Valeriano M. CáceresJr. pelo auxílio em campo, a Michel Miretski pelas sugestões ao manuscrito e à Copel, Capes, CNPq e Curso de Pós-graduação em Zoologia (UFPR) pelo auxílio logístico.

\section{REFERÊNCIAS BIBLIOGRÁFICAS}

ATRAMENTOWICZ, M., 1986, Dynamique de population chez trois marsupiaux didelphidés de Guyane. Biotropica, 18(2): 136-149.

CÁCERES, N. C. \& MONTEIRO-FILHO. E. L. A., 1998, Population dynamics of the common opossum, Didelphis marsupialis (Mammalia, Marsupialia), in southern Brazil. Z. Säugetierk., 63: 169-172.

CATZEFLIS, F., RICHARD-HANSEN, C., FOURNIERCHAMBRILLON, C., LAVERGNE, A. \& VIÉ, J., 1997, Biométrie, reproduction et sympatrie chez Didelphis marsupialis et $D$. albiventris en Guyane française (Didelphidae: Marsupialia). Mammalia, 61(2): 231-243.

CHARLES-DOMINIQUE, P., ATRAMENTOWICZ, M., CHARLES-DOMINIQUE, M., GÉRARD, H., HLADIK, A., HLADIK, C. M. \& PRÉVOST, M. F., 1981, Les mammiferes frugivores arboricoles nocturnes d'une forest guyanaise: interrrelations plantes-animaux. Rev. Ecol. Terre Vie, 35: 341-435.

CHEREM, J. J., GRAIPEL, M. E., MENEZES, M. E. \& SOLDATELI, M., 1996, Observações sobre a biologia do gambá (Didelphis marsupialis) na Ilha de Ratones Grande, Estado de Santa Catarina, Brasil. Biotemas, 9 (2): 47-56.

EMMONS, L. H. \& FEER, F., 1990, Neotropical rainforest mammals a field guide. The University of Chicago Press, Chicago.

FITCH, H. S. \& SANDIDGE, L. L., 1953, Ecology of the opossum on a natural area in northeastern Kansas. Univ. Kansas Publ., Mus. Nat. Hist., 7(2): 305-338.

FONSECA, G. A. B. \& KIERULFF, M. C. M., 1988, Biology and natural history of Brazilian Atlantic forest mammals. Bull. Florida State Mus., Biol. Sci., 34(3): 99-152.
FONSECA, G. A. B., REDFORD, K. H. \& PEREIRA, L. A., 1982, Notes on Didelphis albiventris (Lund, 1841) of Central Brazil. Ciên. Cult., 34(10): 1359-1362.

GARDNER, A. L., 1973, The systematics of the genus Didelphis (Marsupialia: Didelphidae) in North and Middle America. Spec. Publ. Mus., Texas Tech. Univ., 4: 1- 81.

GARDNER, A. L., 1983, Didelphis marsupialis. In: D. H. Janzen (ed.), Costa Rican natural history, The University of Chicago Press, Chicago.

GILMORE, R. M., 1943, Mammalogy in an epidemiological study of jungle yellow fever in Brazil. J. Mammal., 24: 144-162.

HERSHKOVITZ, P., 1992, The South American gracile mouse opossums, genus Gracilinanus Gardner and Creighton, 1989 (Marmosidae, Marsupialia): a taxonomic review with notes on general morphology and relationships. Fieldiana Zool., N.S., 70: 1-56.

HOSSLER, R. J., McNINCH, J. B. \& HARDER, J. D., 1994, Maternal denning behavior and survival of juveniles in opossums in southeastern New York. J. Mammal., 75(1): 60-70.

ISKJAER, C., SLADE, N. A., CHILDS, J. E., GLASS, G. E. \& KORCH, G. W., 1989, Body mass as a measure of body size in small mammals. J. Mammal., 70(3): 662-667.

LEITE, Y. L. R., STALLINGS, J. D. \& COSTA, L. P., 1994, Partição de recursos entre espécies simpátricas de marsupiais na Reserva Biológica Poço das Antas, Rio de Janeiro. Rev. Bras. Biol., 54(3): 525-536.

MAACK, R., 1981, Geografia física do Estado do Paraná. $2^{\mathrm{a}}$ ed., J. Olympio, Rio de Janeiro, 450p.

MONTEIRO-FILHO, E. L. A., 1987, Biologia reprodutiva e espaço domiciliar de Didelphis albiventris em uma área perturbada na região de Campinas, Estado de São Paulo (Mammalia: Marsupialia). Tese de Mestrado, Universidade Estadual de Campinas, Campinas, VI+89p.

MOORS, P. J., 1980, Sexual dimorfism in the body size of mustelids (Carnivora): the roles of food habits and breeding systems. Oikos, 34: 147-158.

PETRIDES, G. A., 1949, Sex and age determination in the opossum. J. Mammal., 30(4): 364-378.

PINE, R. H., DALBY, P. L. \& MATSON, J. O., 1985, Ecology, postnatal development, morphometrics, and taxonomic status of the short tailed opossum, Monodelphis dimidiata, an apparently semelparous annual marsupial. Ann. Carnegie Mus., 54(6): 195-231.

QUIN, D. G., SMITH, A. P. \& NORTON, T. W., 1996, Ecogeographic variation in size and sexual dimorphism in sugar gliders and squirrel gliders (Marsupialia: Petauridae). Austr. J. Zool., 44: 19-45.

RYSER, J., 1992, The mating system and male mating success of the Virginia Opossum (Didelphis virginiana) in Florida. J. Zool. Lond., 228: 127-139.

SHINE, R., 1989, Ecological causes for the evolution of sexual dimorfism: a review of the evidence. Q. Rev. Biol., 64(4): 419-461. 
SUNQUIST, M. E. \& EISENBERG, J. F., 1993, Reproductive strategies of female Didelphis. Bull. Florida Mus. Nat. Hist., Biol. Sci., 36(4): 109-140.

THOMPSON, S. D., 1987, Body size, duration of parental care, and the intrinsic rate of natural increase in eutherian and metatherian mammals. Oecologia (Berlin), 71: 201209.

TYNDALE-BISCOE, C. H. \& MACKENZIE, R. B., 1976, Reproduction in Didelphis marsupialis and D. albiventris in Colombia. J. Mammal., 57(2): 249-265.
VAREJÃO, J. B. M. \& VALLE, C. M. C., 1982, Contribuição ao estudo da distribuição geográfica do gênero Didelphis (Mammalia: Marsupialia) no Estado de Minas Gerais, Brasil. Lundiana, 2: 5-55.

ZAR, J. H., 1984, Biostatistical analysis. 2르. ed., PrenticeHall, Englewood Cliffs, 718p. 